\title{
Concordancia del IMC y la percepción de la imagen corporal en adolescentes de una localidad suburbana de Yucatán
}

\author{
Yolanda Oliva-Peña 1, Manuel Ordóñez-Luna ${ }^{1}$, Andrés Santana-Carvajal 1, Alina Dioné Marín- \\ Cárdenas ${ }^{2}$, Guadalupe Andueza Pech ${ }^{1}$, Iving Alberto Gómez Castillo ${ }^{2}$ \\ ${ }^{1}$ Centro de Investigaciones Regionales "Dr. Hideyo Noguchi", Universidad Autónoma de Yucatán. ${ }^{2}$ Facultad de Me- \\ dicina, Universidad Autónoma de Yucatán
}

\section{RESUMEN}

Introducción. Los problemas de la imagen corporal asociados con los riesgos de trastornos alimenticios entre los adolescentes, en particular, la anorexia nerviosa y la bulimia, son considerados temas de salud emergentes a nivel mundial. Las consecuencias que conllevan son físicas, psicológicas y sociales.

Objetivo. Determinar la concordancia entre el índice de masa corporal y la percepción de la imagen corporal en un grupo de adolescentes.

Materiales y Métodos. Estudio cuantitativo, observacional, transversal realizado en 84 alumnos, $54.1 \%$ hombres y $45.9 \%$, mujeres, con edades entre 10 y 17 años, que cursaban educación básica y secundaria en una localidad suburbana de alta marginación, en Yucatán. Se auto-administró a los estudiantes, el pictograma de Stunkard, para registrar la percepción de la imagen corporal. Para calcular el índice de masa corporal (IMC) se registraron medidas antropométricas de peso y talla, usando una báscula digital marca Tanita, modelo TBC 310 y un estadímetro marca SECA de 2 metros de longitud. Los puntos de corte fueron los establecidos por la Organización Mundial de la Salud.

Resultados. Los hallazgos, respecto a la percepción de la imagen corporal, mostraron una concordancia insignificante $(\mathrm{k}=0.067, \mathrm{p}<$ 0.5 ); en mujeres es baja, con un valor de $\mathrm{k}=.223$; se perciben normales aun cuando presentan bajo peso, mientras que, las que presentan obesidad, subestiman su estado. En cuanto a los hombres, en los casos de obesidad, la imagen y el IMC son coincidentes, aunque, al mismo tiempo, subestiman la percepción corporal en bajo peso.

Palabras Clave: Imágen corporal, índice de masa corporal, adolescencia

\section{ABSTRACT \\ Concordance of the body mass index and the perception of body image in adolescents in Yucatán}

Introduction. Body image problems associated with the risk of eating disorders such as anorexia nervosa and bulimia, are considered emerging health issues among adolescents around the world. These problems have both physical, psychological and social consequences.

Objective. To determine the correlation between body mass index and the body image perception in a group of teenagers.

Material and Methods. A quantitative, observational, cross-sectional study was carried out in 84 students, $54.1 \%$ men and $45.9 \%$ women with an age range between 10 and 17

Autor para correspondencia: Yolanda Oliva-Peña, Centro de Investigaciones Regionales "Dr. Hideyo Noguchi", Universidad Autónoma de Yucatán, Avenida Itzáes No. 490 x 59, C.P. 97000 Centro, Mérida, Yucatán, México. E-mail: opena@correo.uady.mx

Recibido: el 20 de agosto de 2015 Aceptado para publicación: el 9 de noviembre de 2015

Este documento está disponible en http://www.revbiomed.uady.mx/pdf/rb162722.pdf

Vol. 27, No. 2, mayo-agosto de 2016 


\section{Oliva-Peña et al}

years who were attending primary or secondary school education in a suburban town of high marginalization in Yucatan. Self-applicable Stunkard pictogram was used to record the body image perceptions. To calculate the body mass index (BMI) anthropometric measurements of weight and height were recorded, using a Tanita brand digital scale, TBC model and an estadimetro SECA 3102 meters length. The cutoff points were those established by the World Health Organization.

Results. The findings regarding the perception of body image showed no significant concordance $(\mathrm{k}=0.067, \mathrm{p}<0.5)$; for women was a low value of $\mathrm{k}=0.223$, they perceive themselves as normal despite their low weight, and those with obesity underestimate their status. In the group of men, in cases of obesity, image and BMI are coincident while a body perception underestimates low weight.

Conclusion. It is important to take into the nutritional diagnosis, contents of emotional aspects as self-esteem and feelings that manifest teenagers about their body image for comprehensive preventive care.

Keywords: Body image, body mass index, adolescence

\section{INTRODUCCIÓN}

La adolescencia, constituye una etapa de cambios que comprende aspectos físicos, psicológicos y sociales, que se va desarrollando en diversas magnitudes y formas de acuerdo a la genética y el entorno psicosocial. Se experimentan cambios en el desarrollo psicomotor, cognoscitivo, afectivo y social. Existen dos etapas en este periodo de transición, la adolescencia temprana y la tardía. La adolescencia temprana abarca de $\operatorname{los} 10$ a los 14 años de edad y comienzan a manifestarse algunos cambios físicos como aceleración del crecimiento, desarrollo de los órganos sexuales y aparición de las características sexuales secundarias; estos cambios pueden generar tanto ansiedad, como entusiasmo en los adolescentes. Las niñas y los varones cobran mayor conciencia de su género y pueden ajustar su conducta o apariencia a las normas que observan. En ocasiones, pueden resultar víctimas de actos de intimidación o acoso, o participar en ellos, y sentirse confundidos acerca de su propia identidad, personal y sexual.

La adolescencia tardía comprende entre los 15 y los 19 años de edad; para entonces, usualmente, han tenido lugar los cambios físicos más importantes aunque el cuerpo sigue desarrollándose; el cambio físico involucra el incremento del Índice de Masa Corporal (IMC) como expresión de la madurez biológica $(1,2)$.

La adolescencia, ha sido definida en forma amplia por la Organización Panamericana de la Salud (OPS) y atribuye este concepto al grupo de la población comprendida entre los 10 a los 24 años (3); en este rango de edad se registran alrededor de 232 millones de personas que representa el $24,5 \%$ de la población total de la región de las Américas (4). Según datos del Instituto Nacional de Estadística Geografía e Informática (INEGI) 2010, la población comprendida entre 10-19 años representa el $19.9 \%$ de la totalidad de la población mexicana; dividido en $10 \%$ el sexo masculino y $9.9 \%$ el sexo femenino. En Yucatán, la población registrada en 2010 fue 1,955,577 personas; un tercio del total de la población $(33,3 \%)$, es decir 654,860 personas tenía entre 0 y 17 años. El porcentaje de población masculina $(50.9 \%)$ fue ligeramente mayor que la femenina $(49,1 \%) ; 82,1 \%$ de niñas, niños y adolescentes vivían en poblaciones urbanas y 52,4\% fue de origen indígena (5).

La Encuesta Nacional de Salud y Nutrición 2012, informó que en el grupo de adolescentes, $29,9 \%$ reflejaba sobrepeso y con obesidad, $12,2 \%$. La distribución por sexo en 2012, mostró una prevalencia mayor para mujeres $(44,1 \%)$ en comparación con los hombres $(42,7 \%)(6)$.

\section{Revista Biomédica}


IMC y percepción de la imagen corporal adolescente

Esta información mostró la magnitud de la probable insatisfacción con la imagen corporal en este grupo de población y, por tanto, el potencial riesgo de padecer trastornos alimentarios. La presión social a la que se ven sometidos determinados estratos de la población, como son las mujeres jóvenes, con la imposición de un modelo estético de extrema delgadez, hace que la preocupación por la imagen corporal transcienda al mundo de la salud, tanto física, como mental (7).

La imagen corporal es "la imagen que se forma en la mente de nuestro propio cuerpo, es decir, el modo en que nuestro cuerpo se nos manifiesta" (8). Por tanto, la imagen corporal no está, necesariamente, correlacionada con la apariencia física real, siendo claves las actitudes y valoraciones que el individuo hace de su propio cuerpo. La imagen corporal está formada por diferentes componentes; el perceptual (percepción del cuerpo en su totalidad o bien de alguna de sus partes); el cognitivo (valoraciones respecto al cuerpo o una parte de éste); el afectivo (sentimientos o actitudes respecto al cuerpo o a una parte de éste y sentimientos hacia el cuerpo) y el conductual (acciones o comportamientos que se dan a partir de la percepción) $(9,10)$. La imagen corporal no es fija e inamovible, sino que puede ser diferente a lo largo de la vida, teniendo una mayor relevancia durante la infancia y adolescencia (11). Esta última etapa, es considerada de gran vulnerabilidad hacia la apariencia física debido a que, en ésta, se desarrolla el sentido de la identidad y del rol sexual, por consiguiente, es el periodo más sensible para el inicio de la insatisfacción corporal (12).

Aquellos sujetos, que al evaluar sus dimensiones corporales manifiestan juicios valorativos que no coinciden con las dimensiones reales, presentan una alteración de la imagen corporal (13). Cada cultura, a lo largo del tiempo, ha tenido un estereotipo de imagen corporal, como consecuencia de esto, la imagen corporal está influenciada por diferentes aspectos socioculturales, biológicos y ambientales (8). Actualmente, se promueve la belleza del cuerpo como meta para conseguir el éxito social, poniendo énfasis desmedido en la delgadez. Como consecuencia de lo anterior la población en general y, particularmente, los adolescentes brindan extremada importancia a su cuerpo, lo que se manifiesta con el consumo excesivo de productos adelgazantes, dietas poco saludables y conductas que ponen en riesgo la salud $(10,13)$. A través de los medios de comunicación, se difunden modelos ideales de cuerpo, a partir de los cuales las mujeres toman el referente para su satisfacción personal (14).

Dentro de los factores asociados directamente con la presencia de insatisfacción hacia el propio cuerpo, se encuentra el IMC. Las personas más delgadas son las que indican percepción física positiva, mientras que, los que cursan con sobrepeso, manifiestan mayor insatisfacción. El escaso agrado con la apariencia física va asociado también, a una baja autoestima, sentimiento de ineficacia, inseguridad, depresión y ansiedad interpersonal, que hace que la persona se sienta incómoda o inadecuada en sus interacciones sociales y, en general, con una autoevaluación negativa. Además, si se permite que el problema avance, existe riesgo elevado de desarrollar síntomas de trastornos en la alimentación (15).

Por otra parte, el IMC nos sugiere el estado corporal a partir de una escala que establece lo normal frente a lo patológico, utilizadas principalmente, para evaluar el estado nutricional, el riesgo a la salud y el diagnóstico de mala nutrición y obesidad; esta escala, que se refiere al cociente entre la masa corporal en kilogramos y la talla (altura) al cuadrado, constituye un indicador estandarizado que, en un estudio comparativo con la percepción, nos acerca a la comprensión de posibles distorsiones $\mathrm{y}$, en consecuencia, al riesgo de trastornos 


\section{Oliva-Peña et al}

alimentarios.

En las sociedades contemporáneas, se observa un incremento en problemas de salud relacionados con la imagen corporal a partir de la insatisfacción o preocupación corporal excesiva en edades cada vez más tempranas (16). En los últimos años, diversos estudios han sugerido la importancia de la alteración de la percepción de la imagen corporal como un síntoma precoz para la detección de riesgo de trastorno alimentario y depresión, entre otros $(17,18,19)$. A su vez, según lo reportado en la literatura, los adolescentes tienen una particular propensión a padecer trastornos alimentarios, derivados de profundas ansiedades sobre la imagen corporal alentadas por los estereotipos culturales y mediáticos de la belleza (20).

La modificación de los hábitos alimentarios, el realizar dietas y actividad física en busca de conseguir una imagen adecuada a la estética dominante, constituye un problema de salud emergente entre la juventud actual. En las mujeres, uno de los factores que puede jugar un papel en el aumento de la incidencia de la anorexia nerviosa, es la presión cultural del modelo del cuerpo delgado y optan, principalmente, por realizar dietas (21).

El trastorno de la imagen corporal se refiere a la insatisfacción y a la preocupación por el cuerpo que no se adecúan a la realidad, ocupan la mente con intensidad y frecuencia generando malestar e interfiriendo negativamente en la vida cotidiana (22).

La insatisfacción puede referirse a partes del cuerpo o a cuestiones más de forma, de tamaño o de peso, entre otros. Las alteraciones de la imagen corporal incluyen la distorsión perceptiva de la talla y la insatisfacción y/o preocupación por la imagen corporal (23).

Se estima que en el mundo, $1 \%$ de los adolescentes y adultos jóvenes, sufre anorexia y, $4,1 \%$, bulimia. Con relación a los trastornos de la conducta alimentaria (TCA), de 5 a $13 \%$ de la población mundial, presenta síndromes parciales de estas alteraciones. De acuerdo con la OMS, la anorexia inicia en edades que van de los 14 a los 18 años, en tanto que, la bulimia, suele ser más frecuente en mayores de 16 años. Entre los factores de riesgo existentes, se encuentran la preocupación excesiva por el peso, insatisfacción con la figura y una dieta poco saludable, baja autoestima, dificultades para enfrentar el estrés y los conflictos afectivos, e incluso, ser víctima de acoso escolar o bullying, (24).

En México, los casos de anorexia se presentan en $0.1 \%$ de la población de adolescentes que, en su mayoría, afecta a mujeres. En la población juvenil mexicana se estima que la bulimia afecta a 0,4\% (24). En la última década, los estudios sobre imagen corporal en adolescentes han proliferado en distintos contextos y continentes; en cuanto al área hispana, tenemos como referentes los realizados en España; algunos de los hallazgos reportados en estos estudios indican que existe un grado importante de insatisfacción con la imagen corporal y diferencias por sexo; se destaca mayor insatisfacción en las mujeres, pero son los hombres con mayor edad los que encuentran mayor precisión entre el IMC y la percepción de la imagen corporal; asimismo, son las mujeres las que realizan dietas para mejorar su imagen (16,25-31). Perpiñá C, Baños R., en 1990, encontraron en los y las chicas estudiadas una que sobreestiman el tamaño corporal, lo que puede considerarse como un índice de insatisfacción con el propio cuerpo, que está más relacionada con el peso percibido que con el real (31).

En América latina, se han desarrollado diversos trabajos enfocados a distintos grupos de población. En mujeres adultas se muestra que, a menor peso corporal, mayor satisfacción de la imagen corporal (32).

En estudiantes universitarios de Chile y Panamá se halló que más de $60 \%$ de los estudiantes chilenos sobreestimaba su peso y

\section{Revista Biomédica}


IMC y percepción de la imagen corporal adolescente

menos de $20 \%$ se ve como realmente es. Por el contrario, en Panamá se sobreeestima menos de $50 \%$ (hombres $40,8 \%$ y mujeres $37,7 \%$ ), equiparando los resultados de la subestimación del IMC (hombres $38,6 \%$ y mujeres $43,4 \%$ ). En la mitad de los casos hubo concordancia entre ambos criterios, lo que se refleja en un índice Kappa de 0,07, que es considerado un valor bajo. Una mejor concordancia se observó en personas con sobrepeso (69\%), disminuyendo a $29,7 \%$ en personas con normopeso y $0 \%$, en personas obesas. La situación más frecuente es la subestimación del peso especialmente en las personas con normopeso (64\%) cuando se presenta sobrepeso y obesidad, destacando que ninguna de las personas obesas se consideró como tal. Se observa una concordancia diagnóstica de $40 \%$ en estudiantes varones panameños, lo que refleja un índice kappa de 0,04. El 100\% de los estudiantes con normopeso sobreestima su imagen con sobrepeso $82,8 \%$, en cambio, de los estudiantes obesos, $96,1 \%$ subestima su peso (34).

Por otra parte, en Costa Rica, se estudió a un grupo de adolescentes encontrando que, en general, hay diferencias significativas en cuanto al género, $\mathrm{F}(1,532)=35.77, \mathrm{p}<.01$, acerca de la insatisfacción con el físico (ver el físico como un problema). Por una parte, son las mujeres quienes se sienten más insatisfechas con su apariencia. Para las mujeres, las partes del cuerpo las cuales representan un problema (desagradan, incomodan) son el abdomen, la cintura, los muslos, las piernas, las caderas y las nalgas. Los hombres encuentran menos problema con su imagen corporal, aunque hay partes del cuerpo que les preocupan, sobre todo el abdomen, el cutis y el tórax (35).

Estos temas han resultado de interés en México, donde se han realizado estudios mayormente con población urbana y adulta (36- 38,59,40). El estudio sobre obesidad revela que la imagen del cuerpo involucra actitudes, percepciones, imaginaciones, emociones y sensaciones físicas sobre y del cuerpo; la mayoría de las personas entrevistadas afirmaron tener percepciones positivas sobre sí mismas, sin embargo, en la condición civil de solteros (as), el motivo más preocupante de su obesidad es por tener un compañero(a). Su imagen se devalúa a tal grado que, por su condición de obesidad, sienten que no merecen ser amadas. Ello conduce a una inseguridad extrema, baja autoestima y malestar en situaciones sociales (39). En otro estudio realizado en personas mayores de 18 años obesas, los entrevistados no se percibieron como obesos sino sólo como «gorditos o llenitos». Por su aspecto y su forma de comer fueron blancos de reproches, críticas o consejos para bajar de peso; además expresaron sentirse inconformes con su imagen corporal, ya que relacionaron la belleza física con la delgadez. (36).

Entre los estudios efectuados en población joven (41-49) podemos mencionar uno con niñas/os mexicanos de 9 a 12 años en el que se encontró que los estereotipos de delgadez extrema impactan más en las niñas que en los varones, particularmente a los varones de más edad. La comparación por género, mostró en las niñas una menor autoestima general y corporal que en los niños; igualmente, la imagen ideal (cómo quieren ser) y futura (cómo creen que serán) de las niñas fue más delgada (47). En otra investigación sobre obesidad en adolescentes, identificaron como causa principal la genética y tendieron a subestimar la obesidad; asimismo, presentaron baja autoestima. Se concluyó que, si bien la obesidad tiene implicaciones en la salud, también la tiene en los aspectos psicológico y social de quienes la padecen (48).

Otro trabajo, relativo al estado nutricional de adolescentes, encontró que 7,1\% tenía bajo peso; $69,8 \%$ peso normal; $11,6 \%$ sobrepeso y $11,6 \%$, obesidad. El análisis de peso autopercibido reveló que $19 \%$, se consideraba delgada o muy delgada; $56 \%$ creía que su peso era normal y 


\section{Oliva-Peña et al}

$25 \%$ se vio a sí mismo como muy gordo. La concordancia entre el peso autopercibido y el estado nutricional fue de 65\% (kappa $=0,36)$. La subestimación del peso se produjo en $24,9 \%$ de 1 los chicos, frente a $20,3 \%$ de las niñas. La sobreestima se produjo en $15,8 \%$ de las niñas, frente a $8,5 \%$ de los chicos (49).

En Jalisco, se analizó la asociación de la precepción corporal y el comportamiento alimentario de control de peso, con la calidad de vida en adolescentes de ambos sexos, con edades entre 11 y 15 años, pertenecientes a escuelas públicas y privadas, concluyéndose que $12,5 \%$ se percibe con peso menor; $25,6 \%$ con sobreestimación y $61,9 \%$, se identificó en el peso cercano al correcto. No se encontró diferencia significativa por género, entre el IMC real y la percepción del peso corporal. (44)

Por su parte, Meza C. y colaboradores, en Monterrey (2013), analizaron la insatisfacción corporal y encontraron en mujeres menor autoconcepto físico, emocional y baja autoestima (44).

Otro estudio, cuyo objetivo era identificar la asociación entre la insatisfacción con la imagen corporal y el índice de masa corporal en los adolescentes (44,8\% varones y 54,7\% mujeres, edad promedio de $13,6 \mathrm{DE}=.93)$, encontró que $81,8 \%$ no mostró insatisfacción con su imagen corporal; $17,7 \%$ presentó insatisfacción leve y $0,5 \%$ insatisfacción moderada. La prevalencia combinada de sobrepeso y obesidad fue de $32 \%$, con una correlación positiva y significativa entre el índice de masa corporal y la insatisfacción con la imagen corporal ( $\mathrm{rs}=.22, \mathrm{p}=.001)$, esta última fue mayor en mujeres que en hombres ( $U$ $=4335.00, \mathrm{p}<.01)$. No se encontró diferencias significativas en la insatisfacción de acuerdo con la edad $(\mathrm{H}=.31, \mathrm{p}>.05)(47)$.

Hasta el momento, no se encontraron estudios sobre el ámbito suburbano, y en general son escasos los estudios en población adolescente que además se encuentre en alta marginación, por lo que en este estudio observamos el proceso en una comunidad de alta marginación, y condición suburbana, componentes claves en la pregunta que guía la investigación ¿cuál es la concordancia de la percepción de la imagen corporal con el IMC en adolescentes suburbanos marginados?

\section{MATERIALES Y METODOS}

Estudio cuantitativo, observacional, transversal con un universo de 84 alumnos de nivel primaria y secundaria que cumplieron con el criterio de edad de 10 años en adelante, cantidad que corresponde al total de alumnos matriculados en dos centros escolares (básico y secundaria) del ciclo 2013-2014 de una comunidad suburbana de Mérida, Yucatán, México. El 54,1\% de hombres y el 45,9\% mujeres. El rango de edad fue de 10 a 17 años, el 22,4\% al momento de la encuesta tenía entre 10 a 11 años; un 45,9\% de 12 a 13 años, de 14 a 15 años un 28,6\% y por último, de 16 y 17 años el 3\%, con una edad media de 12.7 años, la mediana de 13 años y la moda de 13 años.

La comunidad fue seleccionada por ser de alta marginación y estar en transición entre lo rural y lo urbano, es decir suburbana. Se aplicó a los estudiantes un cuestionario autoadministrado, previo consentimiento informado con los tutores, para recabar información general y sobre la percepción de la imagen corporal con el pictograma de Stunkard validado por Cortés para México con una $(\mathrm{ABC}=0.94)(41)$. La percepción de la imagen corporal (PIC) se obtuvo solicitando al estudiante que seleccionara la figura que, según su percepción, correspondiera a su imagen corporal actual de entre nueve figuras corporales diferentes y numeradas para hombres y mujeres. La silueta seleccionada se calificó de la siguiente manera: la 1: como bajo peso; de la 2 a la 5: normal; de la 6 a la 7: sobrepeso y de la 8 a la 9: obesidad (Figura 1).

Para obtener el IMC los jóvenes fueron pesados y medidos usando una báscula digital marca Tanita, modelo TBC 310 y un estadímetro

\section{Revista Biomédica}


IMC y percepción de la imagen corporal adolescente

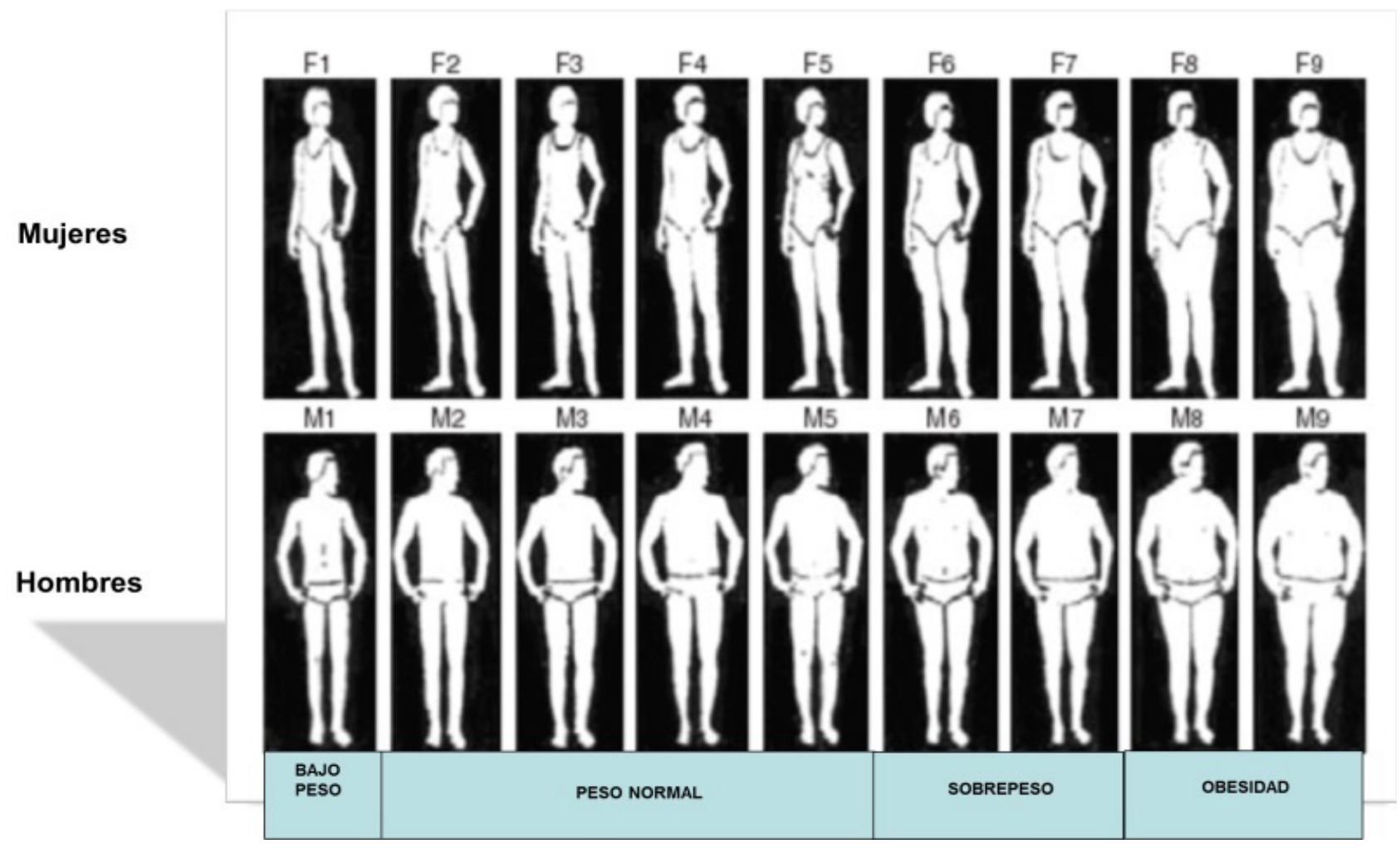

Figura 1. Pictograma Stunkard (Fuente: Stunkard, A \&Album, J (1981)(49)

marca SECA de 2 metros de longitud. Se realizó el cálculo antropométrico; los estudiantes se midieron descalzos, sin prendas en la cabeza ni aditamentos en su ropa. Las mediciones fueron hechas por personal entrenado y estandarizado. Se dividió el peso en kilogramos entre la talla en metros al cuadrado; los puntos de corte para el IMC utilizados fueron los establecidos por la Organización Mundial de la Salud.

Para realizar el procesamiento de la información se empleó el programa SPSS versión 21. Se aplicaron tablas de contingencia para la comparación de prevalencias entre niños y niñas y grupos poblacionales. Para estimar el grado de equivalencia entre las distintas referencias de IMC/edad se calculó el Coeficiente de Kappa. Este coeficiente es una medida de la concordancia que existe entre dos métodos distintos aplicados sobre una misma muestra. Para fines de análisis se crearon tres categorías: bajo peso, normal y obesidad. La valoración del coeficiente kappa se analiza con base en Landis y Koch $(49,50)$.

\section{RESULTADOS}

La talla mínima fue de $1.37 \mathrm{~m}$. y la máxima de $1.70 \mathrm{~m}$. con un promedio de 1.50 $\mathrm{m}$; en cuanto al peso, el promedio fue de 48.1 kilos, el mínimo de 25 kilos y la máxima de 89.2. Se registró $61,9 \%$ en rango normal, seguido de $26,2 \%$ en bajo peso, y $11,9 \%$ en obesidad, la principal problemática en términos absolutos en esta población es el bajo peso y en segundo término la obesidad.

Se registró concordancia entre el IMC real y el percibido en 55,95\% de los casos. 79,8\% de los jóvenes se percibe en normalidad, lo que refleja una diferencia de $17,9 \%$ respecto al IMC; seguido de $26,2 \%$ en bajo peso, con una diferencia a la baja de $19,1 \%$; en cuanto a obesidad, se registra una diferencia al alza de 1,2\%. Se apreció una tendencia de sobreestimación al mismo tiempo que subestimación del peso corporal (Cuadro 1). 
Oliva-Peña et al

Cuadro 1

Distribución porcentual del índice de masa corporal y la percepción corporal

\begin{tabular}{lccc}
\hline Rangos & $\begin{array}{c}\text { Porcentaje } \\
\text { IMC }\end{array}$ & $\begin{array}{c}\text { Porcentaje } \\
\text { percepción } \\
\text { corporal }\end{array}$ & $\begin{array}{c}\text { Diferencia } \\
\text { porcentual }\end{array}$ \\
\hline Bajo peso & 26.2 & 7.1 & -19.1 \\
Normal & 61.9 & 79.8 & +17.9 \\
Obesidad & 11.9 & 13.1 & +1.2 \\
Total & 100 & 100 & \\
\hline
\end{tabular}

El análisis por sexo mostró una concordancia de $41,6 \%$ en las mujeres, quienes tienden a subestimar el bajo peso y la obesidad; mientras que, los hombres subestiman en menor proporción la obesidad, y una concordancia global de 64,5\% Se encontraron diferencias globales por género con una sobrestimación del peso de $29,2 \%$ en las mujeres y una subestimación de $18,4 \%$; en los hombres la sobreestimación fue de $17,7 \%$ y, subestimación, 17,5\%. Los hombres se perciben en mayor porcentaje en condición corporal normal y, en condición de bajo peso, ligeramente mayor a las mujeres (Cuadro 2).

Se indagó sobre comentarios, a propósito de la imagen corporal, entre pares en el ámbito escolar, para observar la presión social sobre la delgadez y otros modelos sociales, encontrando que, uno de cada 5 estudiantes expresó haber recibido comentarios; por sexo, en igual número; por edad, con mayor frecuencia a los 13 años y, en conjunto, $87 \%$ de las menciones fue en el grupo de 12 a 15 años (Cuadro 3).
Otra variable analizada, fue la satisfacción corporal con relación a las partes de su cuerpo que les agradan y las que les desagradan a los adolescentes. Se observó que más de $70 \%$ de hombres y mujeres manifiestó sentirse satisfechos con las partes de su cuerpo, aunque, en los hombres se observó un porcentaje ligeramente mayor. Las partes que mencionan con mayor desagrado para las mujeres están en primer orden el abdomen, la cara, la cadera y los pechos. Mientras que, en los hombres, el abdomen, los brazos y las piernas, lo que concuerda con los estereotipos ideales para lo femenino y lo masculino y el ideal de belleza en la cultura occidental actual.

Por su parte, el análisis de concordancia general, a partir de la prueba kappa que demuestra estadísticamente la fuerza de la concordancia entre la percepción de la imagen corporal y el índice de masa corporal, muestra, una concordancia de fuerza considerable con un valor $(\mathrm{k}=0.067, \mathrm{p}<0.05)$ (Cuadro 4).

El análisis de concordancia por sexo muestra una discordancia no significativa con un valor $\mathrm{k}=-.098$ con una $\mathrm{p}<0.05$ en los hombres $\mathrm{y}$ en las mujeres una concordancia leve con un $\mathrm{k}=$ .223 con una $\mathrm{p}<0.05$, lo que apoya lo hallado en términos porcentuales descrito arriba.

\section{DISCUSIÓN}

Los hallazgos de este estudio demuestran que, la satisfacción de la imagen corporal, concuerda con lo reportado por Trejo y Cols. (46), con un porcentaje mayor de 70\%; diferencia

Cuadro 2

Distribución porcentual del índice de masa corporal y la percepción corporal

\begin{tabular}{lcccccc}
\hline Clasificación & $\begin{array}{c}\text { Percepción } \\
\text { hombres } \\
\%\end{array}$ & $\begin{array}{c}\text { IMC } \\
\text { hombres } \\
\%\end{array}$ & $\begin{array}{c}\text { Diferencia } \\
\%\end{array}$ & $\begin{array}{c}\text { Percepción } \\
\text { mujeres } \\
\%\end{array}$ & $\begin{array}{c}\text { IMC } \\
\text { mujeres } \\
\%\end{array}$ & $\begin{array}{c}\text { Diferencia } \\
\%\end{array}$ \\
\hline Bajo peso & 7.3 & 22.2 & -15.4 & 7 & 25.8 & -18.8 \\
Normal & 78 & 60.3 & +17.7 & 74.4 & 45.2 & +29.2 \\
Obesidad & 14.9 & 17.5 & -2.1 & 18.6 & 29.0 & -10.4 \\
Total & 100 & 100 & 35.5 & 100 & 100 & 58.4 \\
\hline
\end{tabular}

\section{Revista Biomédica}


IMC y percepción de la imagen corporal adolescente

Cuadro 3

Distribución de casos que registraron comentarios sobre su imagen corporal en el ámbito escolar por sexo y edad

\begin{tabular}{lccccccc}
\hline Sexo & \multicolumn{7}{c}{ Edad } \\
\cline { 2 - 8 } & 11 & 12 & 13 & 14 & 15 & 17 & Total \\
\hline Hombre & 1 & 0 & 2 & 3 & 1 & 1 & 8 \\
Mujer & 1 & 3 & 2 & 0 & 2 & 0 & 8 \\
Total & 2 & 3 & 4 & 3 & 3 & 1 & 16 \\
\hline
\end{tabular}

significativa en relación al IMC por sexo, en el que las mujeres registraron peso normal en $45,2 \%$, contra lo observado en hombres, con 60\%. La Encuesta Nacional de Salud y Nutrición (ENSANUT 2012), reporta una prevalencia nacional combinada de sobrepeso y obesidad en adolescentes en $35,8 \%$ en mujeres y $34,1 \%$ en hombres (6). Estos datos nos indican que los resultados de las prevalencias combinadas en nuestro estudio resulta ser un porcentaje inferior en ambos sexos respecto a los datos de la encuesta a nivel nacional.

Sin embargo, es interesante señalar, cómo las mujeres siguen estando con un porcentaje elevado de sobrepeso y obesidad, incluso con una diferencia del peso normal desfavorable en un $45,2 \%$. Esto nos lleva a concluir que existe una tendencia que se dispara en las mujeres adolescentes a enfrentar, en un futuro, las comorbilidades que se derivan de la obesidad.

Nuestros resultados, en cuanto al valor de kappa, coinciden con lo hallado por Durán y Araujo relativos a una baja concordancia; así como la tendencia de los jóvenes, principalmente las mujeres en subestimar de la condición de obesidad $(33,48)$.

Asimismo, el estudio encuentra coincidencias con Salazar en cuanto a las partes del cuerpo que desagradan a hombres y mujeres que demarcan una imagen corporal de acuerdo al estereotipo de género (34).

\author{
Cuadro 4 \\ Distribución de la concordancia general \\ entre el IMC y la percepción \\ de la imagen Corporal
}

\begin{tabular}{lcccc}
\hline IMC & \multicolumn{4}{c}{ Imágen corporal } \\
\cline { 2 - 5 } & Bajo peso & Normal & Obesidad & Total \\
\hline Bajo peso & 3 & 15 & 4 & 22 \\
Normal & 3 & 43 & 6 & 52 \\
Obesidad & 0 & 9 & 1 & 10 \\
Total & 6 & 67 & 11 & 84 \\
\hline
\end{tabular}

De acuerdo a Cabello-Garza, en el contexto de la obesidad, se distinguen dos conceptos relacionados con la imagen corporal: la insatisfacción corporal que es el grado en que los individuos valoran o desprecian su cuerpo, y la distorsión de la imagen corporal, que vendrían a ser la imprecisión en la determinación del tamaño corporal (38). En ese sentido, lo hallado en este estudio respecto a la percepción corporal, es que la distorsión presentada entre la imagen corporal y el IMC en mujeres, es significativa en el caso de las que se perciben normales aún que presenten bajo peso, y subestiman su estado en el caso de las que presentan obesidad. En hombres, coinciden, prácticamente, la imagen y el IMC en los casos de obesidad; al mismo tiempo, expresan una percepción corporal que subestima los casos de bajo peso, lo que nos indica la presencia del modelo de figura de la delgadez aspiracional, ya registrado en las zonas urbanas, como fue encontrado por Montero et.al. y Trejo y Cols. $(45,47)$.

Asimismo, se encontró la presencia de las dos aristas de la malnutrición y/o desnutrición que constituyen posibles factores que naturalizan la delgadez, con un porcentaje de bajo peso de hombres y mujeres superior a lo reportado por Araujo de 7,1\% (49), mientras que lo encontrado en este sector de población es 26,19\%.

A su vez, Ocampo-Barrios y Pérez-Mejía en el estudio a jóvenes con obesidad nos recuerda 
Oliva-Peña et al

que estamos insertos en una cultura que privilegia la imagen corporal estereotipada (35).

Los cambios en los estilos de vida, especialmente la modificación de las conductas alimentarias y de actividad física son la base del tratamiento de la obesidad. Es importante tomar en cuenta las ideas y prácticas realizadas por los adolescentes e incorporar en el diagnóstico nutricio no únicamente contendidos de tipo nutricional sino también considerar elementos de tipo psicológico donde intervienen aspectos emocionales como la autoestima y los sentimientos que se manifiestan por el hecho de considerar tener una imagen corporal diferente a las estereotipadas por la sociedad, lo que permitirá tener una perspectiva más integral.

Así mismo, incluir la perspectiva de género en estos estudios permitirá identificar la situación diferencial que expresan los adolescentes al ser mujeres y hombres en un mundo en el que las zonas rurales y suburbanas se incorporan a la dinámica global, sin embargo con diferencias importantes en la vivencia, atravesadas por la problemática de alta marginación que colocan a este sector de población ante una doble vulnerabilidad de bajo peso y obesidad.

\section{REFERENCIAS}

1. UNICEF. Los derechos de la infancia y la adolescencia en Yucatán [en línea]. UNICEF. 2013. Fecha de acceso Octubre 2015. Disponible en: http://www.unicef.org/ mexico/spanish/SITAN2013_Yucatan.pdf.

2. Burrows R, Díaz N, Muzzo S. Variaciones del índice de masa corporal (IMC) de acuerdo al grado de desarrollo puberal alcanzado. Rev Méd Chile . 2004 Nov; 132 (11): 1363-1368.

3. Organización Panamericana de la Salud. Definición de adolescencia; [ Fecha de acceso: 18 noviembre del 2015] Disponible en: http://www.paho.org/hq/index. php?option $=$ com_content $\&$ view $=$ article $\&$ id $=5173 \&$ Itemid $=2391$

4. UNICEF. Estado Mundial de la Infancia [en línea]. 2011. [Fecha de acceso: 8 de octubre del 2015] Disponible en: http://www.unicef.org/spanish/ sowc2011/pdfs/La-adolenscencia-temprana-y-tardia. pdf

5. Instituto Nacional de Estadística y Geografía. Datos de población y vivienda. Pirámide poblacional 2010. [Fecha de acceso: 8 de octubre del 2015] Disponible en: http://www.inegi.org.mx/sistemas/temasv2/ contenido/DemyPob/epobla28.asp?s=est\&c=17509

6. Instituto Nacional de Salud Pública. Encuesta Nacional de Salud y Nutrición 2012. Disponible en:http://www.insp.mx/produccion-editorial/ novedades-editoriales/3057-ensanut2012-resultadosentidad-federativa.html.

7. Unikel C., Saucedo T., Villatoro J \& Fleiz C. Conductas alimentarias de riesgo y distribución del índice de masa corporal en estudiantes de 13 a 18 años. Salud Mental. 2002 abril; Vol. 25 (2), 49-57.

8. Vaquero-Cristóbal R, Alacid F, Muyor J, LópezMiñarro P. Imagen corporal. Revisión bibliográfica. Nutr Hosp. 2013 Ene-Feb; 28(1):27-35.

9. De la Serna I. Introducción: alteraciones de la imagen corporal. Monog Psiquiatría 2004, 16(2): 1-2.

10. Thompson JK, Heinberg LJ, Altabe M TantleffDunn S Exacting beauty: Theory, assessment, and treatment of body image disturbance. Washington, DC. APA, 1999

11. Raich RM. Imagen Corporal. Conocer y Valorar el Propio Cuerpo. España. Ediciones Pirámide, 2001.

12. García-Fernández L, Garita- Azofeifa E. Relación entre la insatisfacción de la imagen corporal, autoconcepto físico, índice de masa corporal y factores socioculturales en mujeres adolescentes costarricenses. Revista de Ciencias del Ejercicio y la Salud. 2007, 5(1):9-18.

13. Sepúlveda AR, Gandarillas A, Carrobles JA. "Prevalencia de trastornos del comportamiento alimentario en la población universitaria." $5^{\circ}$ Congreso Virtual de Psiquiatría, 2004 Disponible en http:// www.psiquiatria.com/trastornos_de_alimentacion/ prevalencia-de-trastornos-del-comportamientoalimentario-en-la-poblacion-universitaria/.

14. Stice E, Maxfield J, Wells T. Adverse effects of social pressure to be thin on young women: an experimental investigation of the effects of "fat talk". Int J Eat Disord 2003 Jul; 34(1):108-117.

15. Trejo PM, Castro D, Facio A, Mollinedo F, Valdez G. Insatisfacción con la imagen corporal asociada al Índice de Masa Corporal en adolescentes. Revista Cubana de Enfermería. 2010 Mar; 26(3):144-154.

16. Ortega M, Zurita F, González M, Campos B, Ortega A, Torres J. La percepción e insatisfacción corporal en el alumnado de Educación Secundaria de la ciudad de Jaén. Revista en educación. 2013, 11(2): 123-139.

17. Garner DM, Garfinkel PE. Sociocultural factors in the development of anorexia Nervosa. Psychol Mede,

\section{Revista Biomédica}


IMC y percepción de la imagen corporal adolescente

1980, 10 (04):647-656.

18. Hsu LKG. Epidemiology of the eating disorders. Psychiatr Clin North Am, 1996 Dec;19(4):681-700.

19. Unikel C, Mora J, Gómez-Peresmitre G. Percepción de la gordura en adolescentes y su relación con las conductas inapropiadas del comer. Rev Interam Psicol, 1999, 33(1):11-29.

20. Saucedo-Molina TDJ, Unikel C. Conductas alimentarias de riesgo, interiorización del ideal estético de delgadez e índice de masa corporal en estudiantes hidalguenses de preparatoria y licenciatura de una institución privada. Salud Mental. 2010 Enefeb, 33(1): 11-19.

21. Rivarola M F, La imagen corporal en adolescentes mujeres: Su valor predictivo en trastornos alimentarios. Fundamentos en Humanidades 2003IV149-161. Fecha de consulta: 1 de diciembre de 2015 Disponible en: http://www.redalyc.org/articulo.oa?id=18400808

22. Salaberria K, Rodríguez S; Cruz S. Percepción de la imagen corporal. Osasunaz. 2007, 8: 171-183.

23. Phillips, K.A. Body dysmorphic disorder: the distress of imagined ugliness. Am J Psychiatr. 1991 Sep; 148 (9):1138-1149.

24. La jornada. En México aumentan los casos de anorexia y bulimia en hombres. Nota periodística. [Fecha de acceso: 18 de nov. 2015] Disponible en: http://www.jornada.unam.mx/2010/10/25/ sociedad/045n1soc.

25. Ramos P, Rivera F, Moreno M. Diferencias de sexo en imagen corporal, control de peso e índice de masa corporal de los adolescentes españoles. Psicothema. 2010, 22 (1):77-83.

26. Madrigal-Fritsch H, Irala-Estevés, J, MartínezGonzález M, Kearney J, Gibney M; MartínezHernández A. Percepción de la imagen corporal como aproximación al estado de nutrición. Salud pública Mex. 1999 Nov-Dic, 41(6): 479-486.

27. Morrodán M, Montero V, Mesa S, Pacheco J, González M, Bejarano I. Reality, perception and attractiveness of the body image: biological and sociocultural conditions. Zainak. 2008; 30: $15-28$.

28. Ezquerra M. Missperception of the weight in adolescents and relationship with psychological and psychopathological variables. Trastornos de la conducta alimentaria. 2013, 17: 1899-1925.

29. Trujano P, Nava C, de Gracía M, Limón G, Alatriste A, Merino, M. Trastorno de la imagen corporal: Un estudio con preadolescentes y reflexiones desde la perspectiva de género. Anales de psicología. 2010 Jul; 26(2): 279-287.

30. Espina, A, Asunción M, Ochoa I, Yenes F y Alemán A. La imagen corporal en los trastornos alimentarios Psicothema 2001. 13(4): 533-538.
31. Erpiñá C, Baños R. .García L, Garita E. Relación entre la satisfacción con la imagen corporal autoconcepto físico, índice de masa corporal y factores socioculturales en mujeres adolescentes costarricenses. Revista de Ciencias del Ejercicio y la Salud. 2007; 5 (1):9-18.

32. Behar R, Vargas C, Cabrera E. Insatisfacción corporal en los trastornos de la conducta alimentaria: un estudio comparativo. Rev chil neuro-psiquiat 2011 Mar; 49 (1): 26-36.

33. Durán S, Rodríguez M, Record J, Barra R, Olivares $\mathbf{R}$, Tapia A, et al Autopercepción de la imagen corporal en estudiantes universitarios de Chile y Panamá. Rev Chil Nutr. 2013 mar, 40(1): 26-32.

34. Salazar Z. Adolescencia e imagen corporal en la época de la delgadez Reflexiones, 2008, 87(2): 67-80.

35. Ocampo-Barrio P, Pérez-Mejía AN. Creencias y percepciones de las personas obesas acerca de la obesidad. SEMERGEN-Medicina de Familia. 2010 ju-jul; 36(6): 325-331.

36. Osuna-Ramírez I, Hernández-Prado B, Campuzano JC, Salmerón, J. Índice de masa corporal y percepción de la imagen corporal en una población adulta mexicana: la precisión del autorreporte. Salud pública Mex. 2006 Mar-Abril, 48(2): 94-103.

37. Lora-Cortez CI, Saucedo-Molina TDJ. Conductas alimentarias de riesgo e imagen corporal de acuerdo al índice de masa corporal en una muestra de mujeres adultas de la ciudad de México. Salud mental. 2006 May-Jun; 29(3):60-67.

38. Cabello-Garza M. Imagen corporal, percepciones y discursos de aquellos que presentan obesidad media y extrema. Revista de Salud Pública y Nutrición 2011: 2 (12). [Fecha de acceso: 7 octubre del 2015] Disponible en:http://new.medigraphic.com/cgi-bin/resumen.cgi? IDREVISTA $=95 \&$ IDARTICULO $=30343 \&$ IDPUBL ICACION $=3289$.

39. Casillas-Estrella, M; Montaño-Castellón, N; ReyesVázquez, V; Bacardí-Gascón, M; Jiménez-Cruz, A. A mayor IMC mayor grado de insatisfacción de la imagen corporal. Rev Biomed 2006 Oct-Dic 17:14849.

40. Morán I, Cruz L., Iñárritu M. El índice de masa corporal y la imagen corporal percibida como indicadores del estado nutricional en universitarios. Rev Fac Med UNAM 2007 marzo, 50 (2).

41. Cortés-Martínez G, Linet N, Pérez D, Ortiz L. Utilidad de siluetas corporales en la evaluación del estado nutricional en escolares y adolescentes de la Ciudad de México. Bol Med Hosp Infantil Mex. 2009 Nov-dic; 66(6): 511-520.

42. Gómez-Marmol A, Sánchez-Alcaraz BJ, 


\section{Oliva-Peña et al}

Mahadero-Navarrete MP. Insatisfacción y distorsión de la imagen corporal en adolescentes de doce a diecisiete años de edad. Agora for pe and sport. 2013 Ene-Abril; 15(1): 54-63.

43. Hidalgo- Rasmussen CA, Hidalgo-San Martín A. Body weight perception, weight control behavior and quality of life in Mexican adolescents of secondary school. Mexican Journal of Eating Disorders. 2011 Jul-Dic; 2(2):71-81.

44. Meza-Peña C, Pompa-Guajardo E. Body image dissatisfaction in adolescents from Monterrey. International Journal of Good Conscience. 2013 Mar; 8(1): 32-43.

45. Montero P, Morales E, Carbajal A. Evaluation of the perception of body image by the use of anatomical model. Antropo. 2004, 8: 107-116.

46. Trejo P, Castro D, Facio A, Mollinedo F, Valdez, G. Insatisfacción con la imagen corporal asociada al
Índice de Masa Corporal en adolescentes. Revista Cubana de Enfermería. 2010, 26(3):144-154.

47. Martínez-Aguilar M. Percepciones de la obesidad en adolescentes obesos estudiantes del $7^{\circ}$ al $9^{\circ}$ grado residentes de Tamaulipas, México. Rev. Latino Am Enfermagen. 2010 Ene-Feb; 18(1):48-53.

48. Aráujo-Cora L; Dumith S, Menezes A, Hallal P. Measured weight, self-perceived weight, and associates factors in adolescents. Rev Panam Salud Pública 2010 May; 27(5):360-367.

49. Stunkard A, Album J. The accuracy of self-reported weights. Am J Clinic Nutr. 1981 Ago; 34(8):15931599.

50. Cerda J. Villarroel, L. Evaluación de la concordancia inter-observador en investigación pediátrica: coeficiente de Kappa. Rev.Chil. Pediatr. 2008 Feb; 79(1): 54-58.

\section{Revista Biomédica}

